\title{
Asymptotic Completeness for Long-Range Many-Particle Systems with Stark Effect. II
}

\author{
Tadayoshi Adachi ${ }^{1}$, Hideo Tamura ${ }^{2}$ \\ ${ }^{1}$ Department of Mathematical Sciences, University of Tokyo, Meguro-ku, Tokyo 153, Japan \\ ${ }^{2}$ Department of Mathematics, Ibaraki University, Mito, Ibaraki 310, Japan
}

Received: 29 August 1994/in revised form: 19 December 1994

Abstract: We prove the existence and the asymptotic completeness of the Dollardtype modified wave operators for many-particle Stark Hamiltonians with long-range potentials.

\section{Introduction}

The present paper is a continuation to the work [AT] where we have proved the asymptotic completeness of the Graf-type modified wave operators for many-particle Stark Hamiltonians with a class of long-range potentials. We here study the problem of the asymptotic completeness for many-particle Stark Hamiltonians with a larger class of long-range potentials.

We consider a system of $N$ particles moving in a given constant electric field $\mathscr{E} \in \mathbf{R}^{3}, \mathscr{E} \neq 0$. Let $m_{j}, e_{j}$ and $r_{j} \in \mathbf{R}^{3}, 1 \leqq j \leqq N$, denote the mass, charge and position vector of the $j^{\text {th }}$ particle, respectively. The $N$ particles under consideration are supposed to interact with one another through the pair potentials $V_{j k}\left(r_{j}-r_{k}\right), 1 \leqq j<k \leqq N$. Then the total Hamiltonian for such a system is described by

$$
\tilde{H}=\sum_{1 \leqq J \leqq N}\left\{-\frac{1}{2 m_{j}} \Delta_{r_{j}}-e_{j} \mathscr{E} \cdot r_{j}\right\}+V
$$

where $\xi \cdot \eta=\sum_{j=1}^{3} \xi_{j} \eta_{j}$ for $\xi, \eta \in \mathbf{R}^{3}$ and the interaction $V$ is given as the sum of the pair potentials

$$
V=\sum_{1 \leqq j<k \leqq N} V_{j k}\left(r_{j}-r_{k}\right)
$$

As usual, we consider the Hamiltonian $\tilde{H}$ in the center-of-mass frame. We introduce the metric $\langle r, \tilde{r}\rangle=\sum_{j=1}^{N} m_{j} r_{j} \cdot \tilde{r}_{j}$ for $r=\left(r_{1}, \ldots, r_{N}\right)$ and $\tilde{r}=\left(\tilde{r}_{1}, \ldots, \tilde{r}_{N}\right) \in \mathbf{R}^{3 \times N}$. We use the notation $|r|=\langle r, r\rangle^{1 / 2}$. Let $X$ and $X_{\mathrm{cm}}$ be the configuration spaces 
equipped with the metric $\langle\cdot, \cdot\rangle$, which are defined by

$$
\begin{aligned}
X & =\left\{r \in \mathbf{R}^{3 \times N}: \sum_{1 \leqq J \leqq N} m_{J} r_{J}=0\right\}, \\
X_{\mathrm{cm}} & =\left\{r \in \mathbf{R}^{3 \times N}: r_{j}=r_{k} \text { for } 1 \leqq j<k \leqq N\right\} .
\end{aligned}
$$

These two subspaces are mutually orthogonal. We denote by $\pi: \mathbf{R}^{3 \times N} \rightarrow X$ and $\pi_{\mathrm{cm}}: \mathbf{R}^{3 \times N} \rightarrow X_{\mathrm{cm}}$ the orthogonal projections onto $X$ and $X_{\mathrm{cm}}$, respectively. For $r \in \mathbf{R}^{3 \times N}$, we write $x=\pi r$ and $x_{\mathrm{cm}}=\pi_{\mathrm{cm}} r$, respectively. Let $E \in X$ and $E_{\mathrm{cm}} \in X_{\mathrm{cm}}$ be defined by

$$
E=\pi\left(\frac{e_{1}}{m_{1}} \mathscr{E}, \ldots, \frac{e_{N}}{m_{N}} \mathscr{E}\right), \quad E_{\mathrm{cm}}=\pi_{\mathrm{cm}}\left(\frac{e_{1}}{m_{1}} \mathscr{E}, \ldots, \frac{e_{N}}{m_{N}} \mathscr{E}\right)
$$

respectively. Then the total Hamiltonian $\tilde{H}$ is decomposed into $\tilde{H}=H \otimes I d+$ $I d \otimes T_{\mathrm{cm}}$, where $I d$ is the identity operator, $H$ is defined by

$$
H=-\Delta / 2-\langle E, x\rangle+V \quad \text { on } L^{2}(X),
$$

$T_{\mathrm{cm}}$ denotes the free Hamiltonian $T_{\mathrm{cm}}=-\Delta_{\mathrm{cm}} / 2-\left\langle E_{\mathrm{cm}}, x_{\mathrm{cm}}\right\rangle$ acting on $L^{2}\left(X_{\mathrm{cm}}\right)$, and $\Delta$ (resp. $\Delta_{\mathrm{cm}}$ ) is the Laplace-Beltrami operator on $X$ (resp. $\left.X_{\mathrm{cm}}\right)$. We assume that $|E| \neq 0$. This is equivalent to saying that $e_{j} / m_{j} \neq e_{k} / m_{k}$ for at least one pair $(j, k)$. Then $H$ is called an $N$-particle Stark Hamiltonian in the center-of-mass frame.

A non-empty subset of the set $\{1, \ldots, N\}$ is called a cluster. Let $C_{j}, 1 \leqq j \leqq m$, be clusters. If $\bigcup_{1 \leqq j \leqq m} C_{j}=\{1, \ldots, N\}$ and $C_{j} \cap C_{k}=\emptyset$ for $1 \leqq j<k \leqq m, a=$ $\left\{C_{1}, \ldots, C_{m}\right\}$ is called a cluster decomposition. We denote by $\#(a)$ the number of clusters in $a$. We denote by $\tilde{A}$ the set of cluster decompositions and set $\mathscr{A}=$ $\{a \in \tilde{\mathscr{A}}: \#(a) \geqq 2\}$. We let $a, b \in \tilde{\mathscr{A}}$. If $b$ is obtained as a refinement of $a$, that is, if each cluster in $b$ is a subset of a cluster in $a$, we say $b \subset a$, and its negation is denoted by $b \not \subset a$. We note that $a \subset a$ is regarded as a refinement of $a$ itself. If, in particular, $b$ is a strict refinement of $a$, that is, if $b \subset a$ and $b \neq a$, this relation is denoted by $b \subsetneq a$. We denote by $\alpha=(j, k)$ the $(N-1)$-cluster decomposition $\{(j, k),(1), \ldots,(\hat{j}), \ldots,(\hat{k}), \ldots,(N)\}$.

Next we define the two subspaces $X^{a}$ and $X_{a}$ of $X$ as

$$
\begin{aligned}
& X^{a}=\left\{r \in X: \sum_{j \in C} m_{j} r_{j}=0 \text { for each cluster } C \text { in } a\right\}, \\
& X_{a}=\left\{r \in X: r_{j}=r_{k} \text { for each pair } \alpha=(j, k) \subset a\right\} .
\end{aligned}
$$

We note that $X^{\alpha}$ is the configuration space for the relative position of $j^{\text {th }}$ and $k^{\text {th }}$ particles. Hence we can write $V_{\alpha}\left(x^{\alpha}\right)=V_{j k}\left(r_{J}-r_{k}\right)$. These spaces are mutually orthogonal and span the total space $X=X^{a} \oplus X_{a}$, so that $L^{2}(X)$ is decomposed as the tensor product $L^{2}(X)=L^{2}\left(X^{a}\right) \otimes L^{2}\left(X_{a}\right)$. We also denote by $\pi^{a}: X \rightarrow X^{a}$ and $\pi_{a}: X \rightarrow X_{a}$ the orthogonal projections onto $X^{a}$ and $X_{a}$, respectively, and write $x^{a}=\pi^{a} x$ and $x_{a}=\pi_{a} x$ for a generic point $x \in X$. The intercluster interaction $I_{a}$ is defined by

$$
I_{a}(x)=\sum_{\alpha \not \subset a} V_{\alpha}\left(x^{\alpha}\right)
$$


and the cluster Hamiltonian

$$
H_{a}=H-I_{a}=-\Delta / 2-\langle E, x\rangle+V^{a}, \quad V^{a}\left(x^{a}\right)=\sum_{\alpha \subset a} V_{\alpha}\left(x^{\alpha}\right)
$$

governs the motion of the system broken into non-interacting clusters of particles. Let $E^{a}=\pi^{a} E$ and $E_{a}=\pi_{a} E$. Then the operator $H_{a}$ acting on $L^{2}(X)$ is decomposed into

$$
H_{a}=H^{a} \otimes I d+I d \otimes T_{a} \quad \text { on } L^{2}\left(X^{a}\right) \otimes L^{2}\left(X_{a}\right),
$$

where $H^{a}$ is the subsystem Hamiltonian defined by

$$
H^{a}=-\Delta^{a} / 2-\left\langle E^{a}, x^{a}\right\rangle+V^{a} \text { on } L^{2}\left(X^{a}\right),
$$

$T_{a}$ is the free Hamiltonian defined by

$$
T_{a}=-\Delta_{a} / 2-\left\langle E_{a}, x_{a}\right\rangle \text { on } L^{2}\left(X_{a}\right),
$$

and $\Delta^{a}$ (resp. $\Delta_{a}$ ) is the Laplace-Beltrami operator on $X^{a}$ (resp. $X_{a}$ ). By choosing the coordinates system of $X$, which is denoted by $x=\left(x^{a}, x_{a}\right)$, appropriately, we can write $\Delta^{a}=\left|\nabla^{a}\right|^{2}$ and $\Delta_{a}=\left|\nabla_{a}\right|^{2}$, where $\nabla^{a}=\partial_{x^{a}}=\partial / \partial x^{a}$ and $\nabla_{a}=\partial_{x_{a}}=\partial / \partial x_{a}$ are the gradients on $X^{a}$ and $X_{a}$, respectively. We note that we denote by $x^{a}$ (resp. $x_{a}$ ) a vector in $X^{a}$ (resp. $X_{a}$ ) as well as the coordinates system of $X^{a}$ (resp. $X_{a}$ ).

We now state the precise assumption on the pair potentials. Let $c$ be a maximal element of the set $\left\{a \in \mathscr{A}: E^{a}=0\right\}$ with respect to the relation $\subset$. As is easily seen, such a cluster decomposition uniquely exists and it follows that $E^{\alpha}=0$ if $\alpha \subset c$, and $E^{\alpha} \neq 0$ if $\alpha \not \subset c$. Thus the potential $V_{\alpha}$ with $\alpha \not \subset c$ (resp. $\alpha \subset c$ ) describes the pair interaction between two particles with $e_{j} / m_{j} \neq e_{k} / m_{k}$ (resp. $e_{j} / m_{j}=e_{k} / m_{k}$ ). If, in particular, $e_{j} / m_{j} \neq e_{k} / m_{k}$ for any $j \neq k$, then $c$ becomes the $N$-cluster decomposition. We make different assumptions on $V_{\alpha}$ according as $\alpha \not \subset c$ or $\alpha \subset c$. We assume that:

$(V) V_{\alpha}\left(x^{\alpha}\right) \in C^{\infty}\left(X^{\alpha}\right)$ is a real-valued function and has the decay property

$$
\partial_{x^{\alpha}}^{\beta} V_{\alpha}\left(x^{\alpha}\right)=O\left(\left|x^{\alpha}\right|^{-(\rho+|\beta|) / 2}\right) \quad \alpha \not \subset c, \quad \partial_{x^{\alpha}}^{\beta} V_{\alpha}\left(x^{\alpha}\right)=O\left(\left|x^{\alpha}\right|^{-(\rho+|\beta|)}\right) \quad \alpha \subset c
$$

for some $\sqrt{3}-1<\rho \leqq 1$.

Under this assumption, all the Hamiltonians defined above are essentially selfadjoint on $C_{0}^{\infty}$. We denote their closures by the same notations. Throughout the whole exposition, the notations $c$ and $\rho$ are used with the meanings described above. If $V_{\alpha}$ satisfies this decay assumption, then $V_{\alpha}$ is called a long-range potential. To formulate the obtained result precisely, we define the modified wave operators. The definition requires several new notations. We assume that $a \subset c$. Then the subsystem operator $H^{a}$ does not have a uniform electric field, that is, $E^{a}=0$. Hence it may have bound states in $L^{2}\left(X^{a}\right)$. We denote by $P^{a}: L^{2}\left(X^{a}\right) \rightarrow L^{2}\left(X^{a}\right)$ the eigenprojection associated with $H^{a}$. We also denote the direction of $E$ by $\omega=E /|E|$ and write $z=\langle x, \omega\rangle$. We should note that $z=\left\langle x_{a}, \omega\right\rangle$ because of $\omega^{a}=0$. We set $x_{\|}=$ $z \omega$ and $x_{\perp}=x-x_{\|}$, and write $x_{a, \perp}=\pi_{a} x_{\perp}$. Then we can write $x_{a}=\left(x_{a, \perp}, x_{\|}\right)$. We also write $p_{a}=\left(p_{a, \perp}, p_{\|}\right)$for the coordinates dual to $x_{a}=\left(x_{a, \perp}, x_{\|}\right)$and denote by $D_{a}=-i \nabla_{a}=\left(D_{a, \perp}, D_{\|}\right)$the corresponding velocity operator. If we write $\partial_{\|}=\omega \partial_{z}$, we see that $D_{\|}=-i \partial_{\|}$and $D_{a, \perp}=D_{a}-D_{\|}$. Let $I_{a}^{c}$ be the intercluster interaction obtained from $H^{c}$ :

$$
I_{a}^{c}(x)=I_{a}^{c}\left(x^{c}\right)=\sum_{\alpha \subset c, \alpha \not \subset a} V_{\alpha}\left(x^{\alpha}\right) .
$$


We consider the time-dependent Hamiltonian

$$
H_{a D}(t)=H_{a}+I_{a}^{c}\left(t D_{a, \perp}\right)+I_{c}\left(t D_{a, \perp}+t^{2} E / 2\right) \text { on } L^{2}(X) .
$$

Since $D_{a, \perp}$ commutes with $H_{a}$, the three operators on the right-hand side of (1.1) commute with one another. We note that $I_{a}^{c}\left(t D_{a, \perp}\right)=I_{a}^{c}\left(t D_{a}\right)$ for $I_{a}^{c}\left(t p_{a, \perp}\right)=$ $I_{a}^{c}\left(t \pi^{c} p_{a, \perp}\right)=I_{a}^{c}\left(t p_{a}\right)$. Then we denote by $U_{a D}(t)$ the propagator which is generated by $H_{a D}(t)$, that is, $\left\{U_{a D}(t)\right\}_{t \in \mathbf{R}}$ is a family of unitary operators such that for $\psi \in D\left(H_{a D}(0)\right), \psi_{t}=U_{a D}(t) \psi$ is a strong solution of $i d \psi_{t} / d t=H_{a D}(t) \psi_{t}, \psi_{0}=\psi$. $U_{a D}(t)$ is explicitly represented by

$$
U_{a D}(t)=\exp \left(-i t H_{a}\right) \exp \left(-i \int_{0}^{t}\left\{I_{a}^{c}\left(s D_{a, \perp}\right)+I_{c}\left(s D_{a, \perp}+s^{2} E / 2\right)\right\} d s\right) .
$$

With these notations, the Dollard-type modified wave operators in question are now defined by

$$
W_{a D}^{ \pm}=s-\lim _{t \rightarrow \pm \infty} \exp (i t H) U_{a D}(t)\left(P^{a} \otimes I d\right), \quad a \subset c .
$$

It can be easily proved that if these wave operators exist, their ranges are all closed and they have the intertwining property $\exp (i t H) W_{a D}^{ \pm}=W_{a D}^{ \pm} \exp \left(i t H_{a}\right)$ for $t \in \mathbf{R}$.

The main result of this paper is the following theorem.

Theorem 1.1. Assume that $(V)$ is fulfilled. Let $c$ be as above. Then the Dollardtype wave operators $W_{a D}^{ \pm}, a \subset c$, exist, have the intertwining property and are asymptotically complete:

$$
L^{2}(X)=\sum_{a \subset c} \oplus \text { Range } W_{a D}^{ \pm}
$$

If, in particular, $c$ is the $N$-cluster decomposition, that is, no subsystem has zero reduced charge, the asymptotic completeness of the Dollard-type modified wave operators can be also proved under the assumption $(V)$ with $\rho>1 / 2$. For we need not apply the argument of Dereziński [D] to this situation. Furthermore, we can introduce the modifiers which are different from the Dollard-type ones, so that the asymptotic completeness of such modified wave operators can be proved under the assumption $(V)$ with $\rho>0$. This result is an extension of the result for two-particle systems of Jensen-Yajima [JY] and White [W1, W2] to the case of many-particle systems. This problem will be discussed in detail in Sect. 6 .

The problem of the asymptotic completeness for many-particle quantum systems has made great progress for the past several years. For the systems without electric fields, this problem was first solved by Sigal-Soffer [SS1] for a large class of short-range pair potentials. After that work, alternative proofs have been given by several authors (cf. [Grl, Ki, T1, Y and Z]). On the other hand, for the long-range case, Enss [E] first proved the completeness for three-particle systems with the pair potentials decaying like $O\left(\left|x^{\alpha}\right|^{-v}\right)$ at infinity for some $v>\sqrt{3}-1$. This result has been extended by Dereziński [D] and Zielinski [Z] to $N$-particle systems and also the case of potentials decaying more slowly has been dealt with by Gérard [G] and Wang [Wa] for three-particle systems. We should note that the condition $\rho>\sqrt{3}-1$ in our assumption $(V)$ is assumed in order to apply the argument of [D]. 
For the systems with uniform electric fields, if the assumption (V) is satisfied for some $\rho>1, V_{\chi}$ is called a short-range potential. For the class of short-range pair potentials, the ordinary wave operators

$$
W_{a}^{ \pm}=s-\lim _{t \rightarrow \pm \infty} \exp (i t H) \exp \left(-i t H_{a}\right)\left(P^{a} \otimes I d\right)
$$

exist without adding the time-dependent modifiers $I_{a}^{c}\left(t D_{a, \perp}\right)+I_{c}\left(t D_{a, \perp}+t^{2} E / 2\right)$ to the cluster Hamiltonians $H_{a}$. The asymptotic completeness in the short-range case has been proved by Tamura [T3] and Møller [Mø] for $N$-particle systems. However it is known that such wave operators do not generally exist for the class of longrange potentials which we consider here (see [JO] and [O] for the case of twoparticle systems).

In the previous work [AT], we have considered the class of long-range potentials such that

$$
\partial_{x^{\alpha}}^{\beta} V_{\alpha}\left(x^{\alpha}\right)=O\left(\left|x^{\alpha}\right|^{-(\rho+\mu|\beta|)}\right) \quad \alpha \not \subset c,
$$

for some $\rho, \mu>0$ such that $\rho+\mu>1\left(V_{\alpha}\left(x^{\alpha}\right), \alpha \subset c\right.$, satisfy the same assumption as in $(V))$ and we have proved the asymptotic completeness of the Graf-type modified wave operators

$$
W_{a G}^{ \pm}=s-\lim _{t \rightarrow \pm \infty} \exp (i t H) U_{a G}(t)\left(P^{a} \otimes I d\right), \quad a \subset c,
$$

where the propagators $U_{a G}(t)$ are generated by the time-dependent Hamiltonians

$$
H_{a G}(t)=H_{a}+I_{a}^{c}\left(t D_{a}\right)+I_{c}\left(t^{2} E / 2\right),
$$

and are concretely represented by

$$
U_{a G}(t)=\exp \left(-i t H_{a}\right) \exp \left(-i \int_{0}^{t}\left\{I_{a}^{c}\left(s D_{a}\right)+I_{c}\left(s^{2} E / 2\right)\right\} d s\right) .
$$

This type of wave operators was introduced by Graf [Gr2] for two-particle systems (see also [Zo] and [JO]). However it is known that such wave operators do not exist for the class of long-range potentials which we consider here (see [JO] for the case of two-particle systems). Therefore we need introduce the Dollard-type modifiers (1.2).

\section{Preliminaries}

In this section, we recall the known results. First we introduce some notations. We use the following convention for smooth cut-off functions $F$ with $0 \leqq F \leqq 1$, which is often used throughout the discussion below. For sufficiently small $\delta>0$, we define

$$
\begin{array}{llll}
F(s \leqq d)=1 & \text { for } s \leqq d+\delta, & =0 & \text { for } s \geqq d+2 \delta, \\
F(s \geqq d)=1 & \text { for } s \geqq d-\delta, & =0 & \text { for } s \leqq d-2 \delta, \\
F(s=d)=1 & \text { for }|s-d| \leqq \delta, & =0 & \text { for }|s-d| \geqq 2 \delta
\end{array}
$$

and $F\left(d_{1} \leqq s \leqq d_{2}\right)=F\left(s \geqq d_{1}\right) F\left(s \leqq d_{2}\right)$. The choice of $\delta>0$ does not matter to the argument below, but we sometimes write $F_{\delta}$ for $F$ when we want to clarify the dependence on $\delta>0$. 
We define $S_{0}(X)$ by

$$
S_{0}(X)=\left\{q \in C^{\infty}(X):\left|\partial_{x}^{\beta} q(x)\right| \leqq C_{\beta}\langle x\rangle^{-|\beta|}\right\},
$$

where we write $\langle x\rangle=\left(1+|x|^{2}\right)^{1 / 2}$. Let $\omega=E /|E|$ be the direction of $E$. We denote the coordinate $z \in \mathbf{R}$ by $z=\langle x, \omega\rangle$, so that $H$ is written as $H=-\Delta / 2-|E| z+V$. We should note that

$$
\langle z\rangle^{-1 / 2} \nabla(H+i)^{-1},\langle z\rangle^{-1} \nabla \nabla(H+i)^{-1}: L^{2}(X) \rightarrow L^{2}(X)
$$

are bounded.

Now we state the recent result of Herbst and Skibsted [HS], which is concerned with the spectral properties for many-particle Stark Hamiltonians.

Theorem 2.1. Assume that $(V)$ is satisfied. Let $D=-i \nabla$ and $A=\langle\omega, D\rangle=-i \partial_{z}$. Then

(1) H has no bound states.

(2) Let $R>0$ be fixed and let $\Pi: X \rightarrow X$ be an orthogonal projection such that $\Pi E \neq 0$. Then

$$
\left\|F_{\varepsilon}(H=\lambda) F(|\Pi x| \leqq R)\right\| \rightarrow 0, \quad \varepsilon \rightarrow 0,
$$

uniformly in $\lambda \in \mathbf{R}$. In particular, one has

$$
\left\|F_{\varepsilon}(H=\lambda) F\left(\left|x^{\alpha}\right| \leqq R\right)\right\| \rightarrow 0, \quad \varepsilon \rightarrow 0,
$$

for $\alpha \not \subset c$. that

(3) Let $0<\sigma<|E|$. Then one can take $\varepsilon>0$ so small (uniformly in $\lambda \in \mathbf{R}$ )

$$
F_{\varepsilon}(H=\lambda) i[H, A] F_{\varepsilon}(H=\lambda) \geqq \sigma F_{\varepsilon}(H=\lambda)^{2} .
$$

The above theorem plays a basic role in studying the propagation properties of $\exp (-i t H)$. In particular, the uniformity in high energies $\lambda \gg 1$ in the statement $(2)$ is important. This makes it possible to take $A$ as a conjugate operator in the form inequality in (3).

Next we recall the almost analytic extension method due to Helffer and Sjöstrand [HeSj], which is useful in analyzing operators given by functions of self-adjoint operators. For two operators $B_{1}$ and $B_{2}$, we define

$$
\operatorname{ad}_{B_{1}}^{0}\left(B_{2}\right)=B_{2}, \quad \operatorname{ad}_{B_{1}}^{n}\left(B_{2}\right)=\left[\operatorname{ad}_{B_{1}}^{n-1}\left(B_{2}\right), B_{1}\right], \quad n \geqq 1 .
$$

For $m \in \mathbf{R}$, let $\mathscr{F}^{m}$ be the set of functions $f \in C^{\infty}(\mathbf{R})$ such that

$$
\left|f^{(k)}(s)\right| \leqq C_{k}\langle s\rangle^{m-k}, \quad k \geqq 0 \text {. }
$$

If $f \in \mathscr{F}^{m}$ with $m \in \mathbf{R}$, then there exists $\tilde{f} \in C^{\infty}(\mathbf{C})$ such that $\tilde{f}(s)=f(s)$ for $s \in \mathbf{R}, \operatorname{supp} \tilde{f}(\zeta) \subset\{\zeta \in \mathbf{C}:|\operatorname{Im} \zeta| \leqq d(1+|\operatorname{Re} \zeta|)\}$ for some $d>0$ and

$$
\left|\bar{\partial}_{\zeta} \tilde{f}(\zeta)\right| \leqq C_{M}\langle\zeta\rangle^{m-1-M}|\operatorname{Im} \zeta|^{M}, \quad M \geqq 0
$$

Such a function $\tilde{f}(\zeta)$ is called an almost analytic extension of $f$. Let $B$ be a self-adjoint operator. If $f \in \mathscr{F}^{-m}$ with $m>0$, then $f(B)$ is represented by

$$
f(B)=\frac{i}{2 \pi} \int_{\mathbf{C}} \bar{\partial}_{\zeta} \tilde{f}(\zeta)(B-\zeta)^{-1} d \zeta \wedge d \bar{\zeta} .
$$


For $f \in \mathscr{F}^{m}$ with $m \in \mathbf{R}$, we have the following formulas of the asymptotic expansion of the commutator:

$$
\begin{gathered}
{\left[B_{1}, f(B)\right]=\sum_{n=1}^{M-1} \frac{(-1)^{n-1}}{n !} \operatorname{ad}_{B}^{n}\left(B_{1}\right) f^{(n)}(B)+R_{M},} \\
R_{M}=\frac{1}{2 \pi i} \int_{\mathbf{C}} \bar{\partial}_{\zeta} \tilde{f}(\zeta)(B-\zeta)^{-1} \operatorname{ad}_{B}^{M}\left(B_{1}\right)(B-\zeta)^{-M} d \zeta \wedge d \bar{\zeta} .
\end{gathered}
$$

$R_{M}$ is bounded if there exists $k$ such that $m+k<M$ and $\operatorname{ad}_{B}^{M}\left(B_{1}\right)(B+i)^{-k}$ is bounded. For the proof, see [G].

We use the following lemma frequently. For the proof, see [AT].

Lemma 2.2. Let $f_{j} \in C_{0}^{\infty}(\mathbf{R}), 1 \leqq j \leqq 2$, and let $g \in \mathscr{F}^{0}$. Assume that $B$ is a selfadjoint operator such that $\operatorname{ad}_{B}^{j}(H)(H+i)^{-1}, 1 \leqq j \leqq 2$, are bounded from $L^{2}(X)$ into itself. Then

(1) $\left[f_{1}(H), g(B / t)\right]=\left[f_{1}(H), B / t\right] g^{\prime}(B / t)+O\left(t^{-2}\right)$.

(2) $\left[\left[f_{1}(H), B\right], f_{2}(B / t)\right]=O\left(t^{-1}\right)$.

Moreover, let $A$ be as in Theorem 2.1 and $Q=\langle x\rangle$ or $z(=\langle x, \omega\rangle)$. Then

(3) $\left[(H+i)^{-1}, f_{1}(A / t)\right]=O\left(t^{-1}\right),\left[(H+i)^{-1}, f_{1}\left(Q / t^{2}\right)\right]=O\left(t^{-1}\right)$.

(4) $\left[f_{2}(H), f_{1}(A / t)\right]=O\left(t^{-1}\right),\left[f_{2}(H), f_{1}\left(Q / t^{2}\right)\right]=O\left(t^{-1}\right)$.

(5) $\left[Q, f_{1}(A / t)\right]=O\left(t^{-1}\right),\left[f_{2}\left(Q / t^{2}\right), f_{1}(A / t)\right]=O\left(t^{-3}\right)$.

(6) $(H+i)\left[f_{1}(H), f_{2}\left(Q / t^{2}\right)\right]=O\left(t^{-1}\right)$.

Here $O\left(t^{-v}\right)$ denote bounded operators with their norm estimated by $C t^{-v}$ as $t \rightarrow \infty$.

\section{Propagation Properties}

We will prove Theorem 1.1 for the + case only. In this section, we prove some propagation estimates which mean that the solution $\exp (-i t H) \psi$ concentrates asymptotically on classical Stark trajectories as $t \rightarrow \infty$.

We begin by fixing some new notations. We define a conical neighborhood of $\omega=E /|E|$ by $\Gamma\left(\omega, \varepsilon_{1}, r\right)=\left\{x \in X:\langle\omega, x /|x|\rangle \geqq 1-\varepsilon_{1},|x|>r\right\}$ for $\varepsilon_{1}>0$ and $r>0$. We denote by $\mathbf{D}_{H(t)}$ the Heisenberg derivative with respect to the timedependent Hamiltonian $H(t): \mathbf{D}_{H(t)} \Phi(t)=\Phi^{\prime}(t)+i[H(t), \Phi(t)]$. Throughout the discussion below, we always denote by $f \in C_{0}^{\infty}(\mathbf{R})$ a non-negative smooth function with a compact support. We use the notations \|\| and $($,$) for the L^{2}$ norm and scalar product in $L^{2}(X)$, respectively. We also denote by $B(t), t \geqq 1$, operators having the following properties: (1) $f(H) B(t) f(H): L^{2}(X) \rightarrow L^{2}(X)$ is bounded; (2)

$$
\int_{1}^{\infty}|(B(t) f(H) \exp (-i t H) \psi, f(H) \exp (-i t H) \psi)| d t \leqq C\|\psi\|^{2}, \quad \psi \in L^{2}(X) .
$$

First we need the following proposition, which is the same as Proposition 4.2 of [AT]. Thus we omit the proof. 
Proposition 3.1. There exists $M \gg 1$ dependent on $f$ such that:

(1) For $\psi \in L^{2}(X)$,

$$
\int_{1}^{\infty} \frac{d t}{t}\left\|F\left(\langle x\rangle / t^{2}=M\right) f(H) \exp (-i t H) \psi\right\|^{2} \leqq C\|\psi\|^{2} .
$$

(2) For $\psi \in \mathscr{S}(X), \mathscr{S}(X)$ being the Schwartz space over $X$,

$$
\int_{1}^{\infty} \frac{d t}{t}\left\|F\left(\langle x\rangle / t^{2} \geqq M\right) f(H) \exp (-i t H) \psi\right\|^{2}<\infty .
$$

Next we prove the following proposition, which means that the particles are accelerated along the direction $\omega$ with an at least positive acceleration which is less than $|E|$.

Proposition 3.2. Let $0<v<|E|$ and $L>0$. Then for any $\psi \in L^{2}(X)$,

$$
\int_{1}^{\infty} \frac{d t}{t}\left\|F\left(-L \leqq z / t^{2} \leqq v / 2\right) f(H) \exp (-i t H) \psi\right\|^{2} \leqq C\|\psi\|^{2} .
$$

To prove this proposition, we need the two lemmas below. These are proved as Lemmas 4.1 and 4.2 of [AT].

Lemma 3.3. Let $v$ be as above and $K>0$. Then

$$
\int_{1}^{\infty} \frac{d t}{t}\|F(-K \leqq A / t \leqq v) f(H) \exp (-i t H) \psi\|^{2} \leqq C\|\psi\|^{2} .
$$

Lemma 3.4. Let $v$ and $L$ be as above. Then there exists $K \gg 1$ such that

$$
F(A / t \leqq-K) F\left(-L \leqq z / t^{2} \leqq v / 2\right)(H+i)^{-1}=O\left(t^{-1}\right) .
$$

Proof of Lemma 3.3. The proof is done in exactly the same way as in the proof of Lemma 4.1 of [SS2]. Let $G \in \mathscr{F}^{0}$ be defined by

$$
G(s)=\int_{-\infty}^{s} F_{\delta}(-K \leqq u \leqq v)^{2} d u, \quad 0<\delta \ll 1,
$$

so that $G^{\prime}(s)=F_{\delta}(-K \leqq s \leqq v)^{2} \in C_{0}^{\infty}(\mathbf{R})$. We write $f_{\varepsilon, \lambda}(H)=F_{\varepsilon}(H=\lambda)$. We take $0<v+2 \delta<\sigma<|E|$ for $\delta>0$ as above. Then, by Theorem 2.1 (3), there exists $\varepsilon>0$ such that for any $\lambda \in \mathbf{R}$,

$$
f_{\varepsilon, \lambda}(H) i[H, A] f_{\varepsilon, \lambda}(H) \geqq \sigma f_{\varepsilon, \lambda}(H)^{2} .
$$

For such an $\varepsilon>0$, we have only to prove the lemma with $f(H)=f_{\varepsilon, \lambda}(H)$ for any $\lambda \in \mathbf{R}$. We use

$$
\Phi_{1}(t)=G(A / t)
$$

as a propagation observable. We note that $\Phi_{1}(t)$ is uniformly bounded in $t \geqq 1$. The Heisenberg derivative of this observable is calculated as

$$
\mathbf{D}_{H} \Phi_{1}(t)=\Phi_{1}^{\prime}(t)+i\left[H, \Phi_{1}(t)\right] .
$$


If we take $g \in C_{0}^{\infty}(\mathbf{R})$ such that $g=1$ on the support of $f_{\varepsilon, \lambda}$, then

$$
f_{\varepsilon, \lambda}(H) i\left[H, \Phi_{1}(t)\right] f_{\varepsilon, \lambda}(H)=f_{\varepsilon, \lambda}(H) i\left[g(H) H, \Phi_{1}(t)\right] f_{\varepsilon, \lambda}(H) .
$$

By repeated use of Lemma 2.2, we have

$$
f_{\varepsilon, \lambda}(H) i\left[H, \Phi_{1}(t)\right] f_{\varepsilon, \lambda}(H)=F_{1}(t) f_{\varepsilon, \lambda}(H) i[H, A / t] f_{\varepsilon, \lambda}(H) F_{1}(t)+B(t)
$$

with $F_{1}(t)=F_{\delta}(-K \leqq A / t \leqq v)$. Hence it follows from (3.1) that

$$
f_{\varepsilon, \lambda}(H) i\left[H, \Phi_{1}(t)\right] f_{\varepsilon, \lambda}(H) \geqq \sigma t^{-1} F_{1}(t) f_{\varepsilon, \lambda}(H)^{2} F_{1}(t)+B(t) .
$$

On the other hand, the first term on the right side of (3.2) is evaluated as

$$
f_{\varepsilon, \lambda}(H) \Phi_{1}^{\prime}(t) f_{\varepsilon, \lambda}(H) \geqq-(v+2 \delta) t^{-1} f_{\varepsilon, \lambda}(H) F_{1}(t)^{2} f_{\varepsilon, \lambda}(H) .
$$

Thus we obtain

$$
f_{\varepsilon, \lambda}(H) \mathbf{D}_{H} \Phi_{1}(t) f_{\varepsilon, \lambda}(H) \geqq\{\sigma-(v+2 \delta)\} t^{-1} f_{\varepsilon, \lambda}(H) F_{1}(t)^{2} f_{\varepsilon, \lambda}(H)+B(t)
$$

by Lemma 2.2 again. This proves the lemma.

Proof of Lemma 3.4. We set $F_{A}(t)=F(A / t \leqq-K)$ and $F_{z}(t)=F\left(-L \leqq z / t^{2} \leqq\right.$ $v / 2)$. Then $u=(H-i)^{-1} F_{z}(t) F_{A}(t) w$ solves the equation

$$
(H-i) u=F_{z}(t) F_{A}(t) w, \quad w \in L^{2}(X) .
$$

Recall that the conjugate operator $A$ is represented by $A=-i \partial_{z}$. Hence it follows that $A^{2} \leqq-\Delta$ and, by the boundedness of $V$ and the fact that $u$ is the solution of the above equation, we also have $\|A u\| \leqq C\left(\left\|\langle z\rangle^{1 / 2} u\right\|+\|w\|\right)$ with some $C>0$. We set $G_{A}(t)=F(A / t \leqq-K+1)$ and $G_{z}(t)=F\left(-L-1 \leqq z / t^{2} \leqq v / 2+1\right)$. We note that $G_{A}(t) F_{A}(t)=\bar{F}_{A}(t)$ and $G_{z}(t) F_{z}(t)=F_{z}(t)$. Since $A^{2} G_{A}(t) \geqq(K-2)^{2} t^{2} G_{A}(t)$ and $\langle z\rangle G_{z}(t) \leqq \kappa^{2} t^{2} G_{z}(t)$ with $\kappa=\max (L, v / 2)+2$ for $t \geqq 1$, we make repeated use of Lemma 2.2 to obtain that

$$
\begin{gathered}
\|A u\| \geqq\{(K-2) / 2\} t\left\|(H-i)^{-1} F_{z}(t) F_{A}(t) w\right\|-C\|w\|, \\
\left\|\langle z\rangle^{1 / 2} u\right\| \leqq 2 \kappa t\left\|(H-i)^{-1} F_{z}(t) F_{A}(t) w\right\|+C\|w\|
\end{gathered}
$$

for some $C>0$ independent of $t \geqq 1$. Hence we can take $K>0$ so large that $F_{A}(t) F_{z}(t)(H+i)^{-1}=O\left(t^{-1}\right)$. This completes the proof.

Proof of Proposition 3.2. The proof is done in exactly the same way as in the proof of Theorem 4.2 of [SS2]. Let $G \in \mathscr{F}^{0}$ be defined by

$$
G(s)=\int_{-\infty}^{s} F_{\delta}(-L \leqq u \leqq v / 2)^{2} d u,
$$

so that $G^{\prime}(s)=F_{\delta}(-L \leqq s \leqq v / 2)^{2} \in C_{0}^{\infty}(\mathbf{R})$. We set $\Phi(t)=G\left(z / t^{2}\right)$ as a propagation observable. We note that $\Phi(t)$ is uniformly bounded in $t \geqq 1$. We write $F_{1}(t)=F_{\delta}\left(-L \leqq z / t^{2} \leqq v / 2\right)$. Then the Heisenberg derivative of this observable is 
calculated as

$$
\begin{aligned}
\mathbf{D}_{H} \Phi(t) & =t^{-1}\left(A / t-2 z / t^{2}\right) F_{1}(t)^{2}+O\left(t^{-4}\right) \\
& \geqq t^{-1}\left\{v^{\prime}+\delta+\left(A / t-v^{\prime}-\delta\right) F_{\delta}\left(A / t \leqq v^{\prime}\right)^{2}-v-4 \delta\right\} F_{1}(t)^{2}+O\left(t^{-4}\right) \\
& =t^{-1}\left(v^{\prime}-v-3 \delta\right) F_{1}(t)^{2}+R(t) .
\end{aligned}
$$

Here

$$
R(t)=t^{-1}\left(A / t-v^{\prime}-\delta\right) F_{\delta}\left(A / t \leqq v^{\prime}\right)^{2} F_{1}(t)^{2}+O\left(t^{-4}\right),
$$

and we used the fact that $A / t \geqq v^{\prime}+\delta+\left(A / t-v^{\prime}-\delta\right) F_{\delta}\left(A / t \leqq v^{\prime}\right)^{2}$. We take $\delta>0$ so small that $v^{\prime}-v-3 \delta>0$. We shall show that $R(t)=B(t)$, which implies the proposition by virtue of (3.3). Let $g \in C_{0}^{\infty}(\mathbf{R})$ be such that $g=1$ on the support of $f$. By repeated use of Lemma 2.2, we compute

$$
g(H) R(t) g(H)=t^{-1} g(H)\left(A / t-v^{\prime}-\delta\right) F_{1}(t) g(H) F_{1}(t) F_{\delta}\left(A / t \leqq v^{\prime}\right)^{2}+O\left(t^{-2}\right) .
$$

Finally, by Lemma 3.4 and repeated use of Lemma 2.2, we have

$$
\begin{aligned}
g(H) R(t) g(H) & =t^{-1} g(H)\left(A / t-v^{\prime}-\delta\right) F_{1}(t) g(H) F_{1}(t) F_{\delta}\left(-K \leqq A / t \leqq v^{\prime}\right)^{2}+O\left(t^{-2}\right) \\
& =t^{-1} F_{\delta}\left(-K \leqq A / t \leqq v^{\prime}\right) B_{1}(t) F_{\delta}\left(-K \leqq A / t \leqq v^{\prime}\right)+O\left(t^{-2}\right),
\end{aligned}
$$

where $B_{1}(t)$ is uniformly bounded in $t \geqq 1$. By Lemma 3.3, we see that $R(t)=$ $B(t)$.

The next proposition is the most important propagation estimate, which means that the particles asymptotically concentrate in any conical neighborhood of $\omega$.

Proposition 3.5. Let $M$ be as in Proposition 3.1 and $v$ be as in Proposition 3.2. Fix $\varepsilon_{1}>0$ and $r>0$. Assume that $q \in S_{0}(X)$ vanishes in $\Gamma\left(\omega, \varepsilon_{1}, r\right)$. Then

$$
\int_{1}^{\infty} \frac{d t}{t}\left\|F\left(z / t^{2} \geqq v / 2\right) F\left(\langle x\rangle / t^{2} \leqq M\right) q f(H) \exp (-i t H) \psi\right\|^{2} \leqq C\|\psi\|^{2} .
$$

To prove the above proposition, we have only to prove the following lemma. The proof is essentially the same as that of Proposition 7.3 of [T2] (see also [Mø] and $[\mathrm{A}])$.

Lemma 3.6. Let $\tilde{q}(x)=(\langle x\rangle-z)\langle x\rangle^{-1}$. Let $M$ be as in Proposition 3.1 and $v$ be as in Proposition 3.2. Then

$$
\int_{1}^{\infty} \frac{d t}{t}\left\|F\left(z / t^{2} \geqq v / 2\right) F\left(\langle x\rangle / t^{2} \leqq M\right) \tilde{q} f(H) \exp (-i t H) \psi\right\|^{2} \leqq C\|\psi\|^{2} .
$$

Proof. We write $f_{\varepsilon, \lambda}(H)=F_{\varepsilon}(H=\lambda)$. For some $\varepsilon>0$, we have only to prove the lemma with $f(H)=f_{\varepsilon, \lambda}(H)$ for any $\lambda \in \mathbf{R}$. Let $A_{1}=\langle x\rangle^{-1 / 4} A\langle x\rangle^{-1 / 4}$. We write $F(t)=F\left(z / t^{2} \geqq v / 2\right) F\left(\langle x\rangle / t^{2} \leqq M\right)$ and take

$$
\Phi(t)=f_{\varepsilon, \lambda}(H) F(t) A_{1} F(t) f_{\varepsilon, \lambda}(H)
$$

as a propagation observable. We note that $\Phi(t)$ is uniformly bounded in $t \geqq 1$. The Heisenberg derivative of $\Phi(t)$ takes the form

$$
\mathbf{D}_{H} \Phi(t)=f_{\varepsilon, \lambda}(H) F(t) i\left[H, A_{1}\right] F(t) f_{\varepsilon, \lambda}(H)+B(t)
$$


by Propositions 3.1 and 3.2. By a simple computation, we have

$$
i\left[H, A_{1}\right] \geqq|E|\langle x\rangle^{-1 / 2} \tilde{q}-\langle x\rangle^{-1 / 2}\langle\omega, \nabla V\rangle+\langle x\rangle^{-3 / 4}(V-H)\langle x\rangle^{-3 / 4} .
$$

Let $g \in C_{0}^{\infty}(\mathbf{R})$ be such that $g=1$ on the support of $f_{\varepsilon, \lambda}$. By repeated use of Lemma 2.2, we have

$$
\begin{aligned}
& f_{\varepsilon, \lambda}(H) F(t)\langle x\rangle^{-3 / 4}(V-H)\langle x\rangle^{-3 / 4} F(t) f_{\varepsilon, \lambda}(H) \\
& \quad=f_{\varepsilon, \lambda}(H) F(t)\langle x\rangle^{-3 / 4}(V-H) g(H)\langle x\rangle^{-3 / 4} F(t) f_{\varepsilon, \lambda}(H)+O\left(t^{-4}\right) \\
& \quad=O\left(t^{-3}\right) .
\end{aligned}
$$

Next we consider the second term of the right-hand side of (3.5). We take $q \in S_{0}(X)$ such that $q=1$ in $\Gamma\left(\omega, \varepsilon_{1}, 2 r\right)$ and $q=0$ outside $\Gamma\left(\omega, 2 \varepsilon_{1}, r\right)$ for $0<\varepsilon_{1} \ll 1$ and $r>0$. We should note that we can take $\varepsilon_{1}>0$ so small that $\left|\langle x\rangle^{-1 / 2}\langle\omega, \nabla V\rangle\right| \leqq$ $C\langle x\rangle^{-1-\rho / 2}$ on the support of $q$. We set $\bar{q}=1-q \in S_{0}(X)$ and rewrite

$$
f_{\varepsilon, \lambda}(H) F(t)\langle x\rangle^{-1 / 2}\langle w, \nabla V\rangle F(t) f_{\varepsilon, \lambda}(H)=T_{1}+T_{2},
$$

where

$$
\begin{aligned}
& T_{1}=f_{\varepsilon, \lambda}(H) F(t)\langle x\rangle^{-1 / 2}\langle\omega, \nabla V\rangle q F(t) f_{\varepsilon, \lambda}(H), \\
& T_{2}=f_{\varepsilon, \lambda}(H) F(t)\langle x\rangle^{-1 / 2}\langle\omega, \nabla V\rangle \bar{q} F(t) f_{\varepsilon, \lambda}(H) .
\end{aligned}
$$

It is easy to see that $T_{1}=O\left(t^{-2-\rho}\right)$. We write $h=\langle\omega, \nabla V\rangle \bar{q} / \tilde{q}$. We should note that $\tilde{q} \geqq c>0$ on the support of $\bar{q}$. We set $g_{\varepsilon, \lambda}(H)=F_{2 \varepsilon}(H=\lambda)$, so that $g_{\varepsilon, \lambda}(H) f_{\varepsilon, \lambda}(H)=f_{\varepsilon, \lambda}(H)$. Then, by Lemma 2.2, we get

$$
T_{2}=f_{\varepsilon, \lambda}(H) F(t)\langle x\rangle^{-1 / 2} \tilde{q} h g_{\varepsilon, \lambda}(H) F(t) f_{\varepsilon, \lambda}(H)+O\left(t^{-2}\right) .
$$

We denote $M^{\prime}=\#\{\alpha: \alpha \not \subset c\}$. Then we see that we can take $R>0$ so large that for any $\alpha \not \subset c$,

$$
\left\|\left\{1-F\left(\left|x^{\alpha}\right| \leqq R\right)\right\}\left\langle\omega, \nabla V_{\alpha}\right\rangle(\bar{q} / \tilde{q})\right\| \leqq|E| /\left(4 M^{\prime}\right) .
$$

On the other hand, by Theorem 2.1 (2), we see that we can take $\varepsilon>0$ so small that for any $\alpha \not \subset c$ and $\lambda \in \mathbf{R}$,

$$
\left\|F\left(\left|x^{\alpha}\right| \leqq R\right)\left\langle\omega, \nabla V_{\alpha}\right\rangle(\bar{q} / \tilde{q}) g_{\varepsilon, \lambda}(H)\right\| \leqq|E| /\left(4 M^{\prime}\right) .
$$

Hence, for such an $\varepsilon>0$, we have

$$
\left\|h g_{\varepsilon, \lambda}(H)\right\| \leqq|E| / 2
$$

and

$$
T_{2} \leqq(|E| / 2) f_{\varepsilon, \lambda}(H) F(t)\langle x\rangle^{-1 / 2} \tilde{q} F(t) f_{\varepsilon, \lambda}(H)+B(t) .
$$

Combining this fact with (3.4), (3.5) and (3.6), we obtain

$$
\begin{aligned}
\mathbf{D}_{H} \Phi(t) & \geqq(|E| / 2) f(H) F(t)\langle x\rangle^{-1 / 2} \tilde{q} F(t) f(H)+B(t) \\
& \geqq(|E| / 2)(M+1)^{-1 / 2} t^{-1} f(H) F(t) \tilde{q} F(t) f(H)+B(t),
\end{aligned}
$$

which implies the lemma.

By the above propositions, we conclude the following propagation properties. The proof is similar to that of Lemma 4.5 of [AT]. We omit the proof. 
Proposition 3.7. Let $M, v$ and $q \in S_{0}(X)$ be as above. Let $\Phi(t)$ denote one of the following three operators:

$$
F\left(\langle x\rangle / t^{2} \geqq M\right), \quad F\left(z / t^{2} \leqq v / 2\right), \quad F\left(z / t^{2} \geqq v / 2\right) F\left(\langle x\rangle / t^{2} \leqq M\right) q .
$$

Then

$$
s-\lim _{t \rightarrow \infty} \Phi(t) f(H) \exp (-i t H)=0
$$

\section{Time-Dependent Hamiltonians}

In this section, we introduce an auxiliary time-dependent Hamiltonian which approximates the full Hamiltonian $H$ and study the relation between $\exp (-i t H)$ and the propagator generated by such a time-dependent Hamiltonian.

Let $q_{c} \in S_{0}(X)$ be such that $q_{c}=1$ in $\Gamma\left(\omega, \varepsilon_{1},|E| / 3\right)$, and $q_{c}=0$ outside $\Gamma\left(\omega, 2 \varepsilon_{1},|E| / 4\right)$. Let $\tilde{q}_{c} \in S_{0}(X)$ be such that $\tilde{q}_{c}=1$ in $\Gamma\left(\omega, 2 \varepsilon_{1},|E| / 4\right)$, and $\tilde{q}_{c}=0$ outside $\Gamma\left(\omega, 3 \varepsilon_{1},|E| / 5\right)$. By definition, it follows that $\tilde{q}_{c} q_{c}=q_{c}$. We define

$$
\begin{aligned}
& \varphi_{c}(t, x)=F\left(\langle x\rangle / t^{2} \leqq M\right) F\left(z / t^{2} \geqq|E| / 3\right) q_{c}(x), \\
& W_{c}(t, x)=W_{c}\left(t, x^{c}, x_{c}\right)=F\left(z / t^{2} \geqq|E| / 4\right) \tilde{q}_{c}(x) I_{c}(x) .
\end{aligned}
$$

We should note that $\varphi_{c}(t, x) I_{c}(x)=\varphi_{c}(t, x) W_{c}(t, x)$. By assumption, $W_{c}$ obeys the estimate

$$
\left|\partial_{t}^{m} \partial_{x}^{\beta} W_{c}(t, x)\right| \leqq C_{m \beta}\langle t\rangle^{-m}\left(\langle t\rangle+\langle x\rangle^{1 / 2}\right)^{-\rho-|\beta|} .
$$

We consider the time-dependent Hamiltonian

$$
H_{c}(t)=H_{c}+W_{c}(t), \quad W_{c}(t)=W_{c}(t, x),
$$

and denote by $U_{c}(t)$ the propagator generated by $H_{c}(t)$, that is, $\left\{U_{c}(t)\right\}_{t \geqq 1}$ is a family of unitary operators such that for $\psi \in D\left(H_{c}(1)\right), \psi_{t}=U_{c}(t) \psi$ is a strong solution of $i d \psi_{t} / d t=H_{c}(t) \psi_{t}, \psi_{1}=\psi$.

By the almost analytic extension method, we see that for $f \in C_{0}^{\infty}(\mathbf{R})$,

$$
\mathbf{D}_{H_{c}(t)} f\left(H_{c}(t)\right)=\frac{d}{d t}\left\{f\left(H_{c}(t)\right)\right\}=O\left(t^{-1-\rho}\right),
$$

due to (4.3). By virtue of this estimate (4.4), we obtain the analogue of Propositions 3.1,3.2,3.5 and 3.7. Since the proofs are similar to those of theirs, we omit the proofs.

Proposition 4.1. There exists $M \gg 1$ dependent on $f$ such that:

(1) For $\psi \in L^{2}(X)$,

$$
\int_{1}^{\infty} \frac{d t}{t}\left\|F\left(\langle x\rangle / t^{2}=M\right) f\left(H_{c}(t)\right) U_{c}(t) \psi\right\|^{2} \leqq C\|\psi\|^{2} .
$$

(2) For $\psi \in \mathscr{S}(X), \mathscr{S}(X)$ being the Schwartz space over $X$,

$$
\int_{1}^{\infty} \frac{d t}{t}\left\|F\left(\langle x\rangle / t^{2} \geqq M\right) f\left(H_{c}(t)\right) U_{c}(t) \psi\right\|^{2}<\infty .
$$


Proposition 4.2. Let $0<v<|E|$ and $L>0$. Then for any $\psi \in L^{2}(X)$,

$$
\int_{1}^{\infty} \frac{d t}{t}\left\|F\left(-L \leqq z / t^{2} \leqq v / 2\right) f\left(H_{c}(t)\right) U_{c}(t) \psi\right\|^{2} \leqq C\|\psi\|^{2} .
$$

Proposition 4.3. Let $M$ be as Proposition 4.1 and $v$ be as Proposition 4.2. Fix $\varepsilon_{1}>0$ and $r>0$. Assume that $q \in S_{0}(X)$ vanishes in $\Gamma\left(\omega, \varepsilon_{1}, r\right)$. Then

$$
\int_{1}^{\infty} \frac{d t}{t}\left\|F\left(z / t^{2} \geqq v / 2\right) F\left(\langle x\rangle / t^{2} \leqq M\right) q f\left(H_{c}(t)\right) U_{c}(t) \psi\right\|^{2} \leqq C\|\psi\|^{2} .
$$

Proposition 4.4. Let $M, v$ and $q \in S_{0}(X)$ be as above. Let $\Phi(t)$ denote one of the following three operators:

$$
F\left(\langle x\rangle / t^{2} \geqq M\right), \quad F\left(z / t^{2} \leqq v / 2\right), \quad F\left(z / t^{2} \geqq v / 2\right) F\left(\langle x\rangle / t^{2} \leqq M\right) q .
$$

Then

$$
s-\lim _{t \rightarrow \infty} \Phi(t) f\left(H_{c}(t)\right) U_{c}(t)=0 .
$$

Next we show the existence of the following two strong limits, the first limit being often called the Deift-Simon wave operator (see [Grl, SS1, Z and A]).

Theorem 4.5. Let the notations be as above. Then there exist the following strong limits:

$$
s-\lim _{t \rightarrow \infty} U_{c}(t)^{*} \exp (-i t H), \quad s-\lim _{t \rightarrow \infty} \exp (i t H) U_{c}(t)
$$

Proof. The proof for the existence of the first limit is similar to that of Lemma 5.2 of [AT]. By Proposition 3.7, we have only to prove the existence of the strong limit

$$
s-\lim _{t \rightarrow \infty} U_{c}(t)^{*} \varphi_{c}(t, x) f(H) \exp (-i t H) .
$$

Taking $f_{1} \in C_{0}^{\infty}(\mathbf{R})$ such that $f_{1} f=f$ and noting $\varphi_{c}(t, x)\left(W_{c}(t, x)-I_{c}(x)\right)=0$, we see that

$$
f_{1}\left(H_{c}(t)\right) \varphi_{c}(t, x)-\varphi_{c}(t, x) f_{1}(H)=O\left(t^{-1}\right),
$$

by virtue of the almost analytic extension method. From this fact, the existence of (4.5) can be proved by the same argument of [AT].

Next we note that for $\varepsilon>0$ small enough and $\psi \in L^{2}(X)$, there exists $f \in$ $C_{0}^{\infty}(\mathbf{R})$ such that

$$
\left\|\left\{I d-f\left(H_{c}(t)\right)\right\} U_{c}(t) \psi\right\|=O(\varepsilon)
$$

uniformly in $t \geqq 1$, which is proved by virtue of (4.4). Now we take $M$ as in Proposition 4.1 for this $f$ and define $\varphi_{c}(t, x)$ by (4.1) with this $M$. Then, by virtue of Proposition 4.4, we have only to prove the existence of the strong limit

$$
s-\lim _{t \rightarrow \infty} \exp (i t H) \varphi_{c}(t, x) f\left(H_{c}(t)\right) U_{c}(t),
$$

which may be proved in the same way as that of (4.5).

By the existence of the first strong limit in the above proposition, we obtain the following theorem immediately. 
Theorem 4.6 (Asymptotic clustering). Let the notation be as above. Then for $\psi \in L^{2}(X)$, there exists $\psi_{c} \in L^{2}(X)$ such that

$$
\exp (-i t H) \psi=U_{c}(t) \psi_{c}+o(1) .
$$

\section{Proof of Theorem 1.1}

In this section, we complete the proof of Theorem 1.1. By Theorem 4.6, the proof is now reduced to studying the propagation properties of the propagator $U_{c}(t)$ generated by the time-dependent Hamiltonian $H_{c}(t)$. For this sake, we introduce a family of the unitary operators $\{T(t)\}_{t \in \mathbf{R}}$ on $L^{2}(X)$ as follows: For $u(x) \in L^{2}(X)$, we define

$$
(T(t) u)(x)=\exp \left(i t|E| z-i t^{3}|E|^{2} / 6\right) u\left(x-t^{2} E / 2\right) .
$$

We also introduce the time-dependent Hamiltonians

$$
\begin{gathered}
\tilde{H}_{a D}(t)=H_{a}+I_{a}^{c}\left(t D_{a}\right)+I_{c}\left(t D_{a}-t^{2} E / 2\right), \\
H_{a, S 1}(t)=H_{a}+I_{a}^{c}\left(t D_{a}\right)+W_{c}\left(t, 0, t D_{a}-t^{2} E / 2\right), \\
H_{c, M 0}(t)=H_{c, M}+W_{c}\left(t, x^{c}, x_{c}+t^{2} E / 2\right), \\
H_{a, M 1}(t)=H_{a, M}+I_{a}^{c}\left(t D_{a}\right)+W_{c}\left(t, 0, t D_{a}+t^{2} E / 2\right),
\end{gathered}
$$

for $a \subset c$, where $H_{a, M}=-\Delta / 2+V^{a}\left(x^{a}\right)$ acts on $L^{2}(X)$ and has no electric fields. We denote by $\tilde{U}_{a D}(t), U_{a, S 1}(t), U_{c, M 0}(t)$ and $U_{a, M 1}(t)$ the propagators generated by $\tilde{H}_{a D}(t), H_{a, S 1}(t), H_{c, M 0}(t)$ and $H_{a, M 1}(t)$, respectively, where $\tilde{U}_{a D}(0)=I d, U_{a, S 1}(1)=$ $I d, U_{c, M 0}(1)=I d$ and $U_{a, M 1}(1)=I d$. Since $\exp \left(-i t H_{a}\right) D_{a} \exp \left(i t H_{a}\right)=D_{a}-t E$ for $a \subset c, \tilde{U}_{a D}(t)$ is explicitly represented by

$$
\tilde{U}_{a D}(t)=\exp \left(-i t H_{a}\right) \exp \left(-i \int_{0}^{t}\left\{I_{a}^{c}\left(s D_{a}\right)+I_{c}\left(s D_{a}+s^{2} E / 2\right)\right\} d s\right) .
$$

The family of transformations $\{T(t)\}_{t \in \mathbf{R}}$ was introduced by Jensen-Yajima [JY], by which Stark Hamiltonians are transformed into Hamiltonians without electric fields (see also $[\mathrm{AH}]$ ). In fact, we see by the argument similar to [JY] that

$$
U_{c}(t)=T(t) U_{c, M 0}(t) T(1)^{-1}, \quad U_{a, S 1}(t)=T(t) U_{a, M 1}(t) T(1)^{-1} .
$$

We should note that $V^{a}\left(x^{a}\right)$ in $H_{a, M}$ does not undergo a change under the transformation $T(t)$. By virtue of the relation (5.2), we have only to study the asymptotic behavior of the propagator $U_{c, M 0}(t)$ generated by the time-dependent Hamiltonian $H_{c, M 0}$. We now apply to $H_{c, M 0}$ the result due to Dereziński [D] on the asymptotic completeness for long-range many-particle quantum systems without electric fields (see also [Z]).

Proposition 5.1. Assume that $(V)$ is fulfilled. Then the wave operators

$$
\Omega_{a, M 1}=s-\lim _{t \rightarrow \infty} U_{c, M 0}(t)^{*} U_{a, M 1}(t)\left(P^{a} \otimes I d\right)
$$


exist for all $a \subset c$, and are asymptotically complete, that is,

$$
L^{2}(X)=\sum_{a \subset c} \oplus \text { Range } \Omega_{a, M 1} .
$$

The condition $\rho>\sqrt{3}-1$ in the assumption $(V)$ is essentially used to prove this proposition only. We go back to the original propagator $U_{c}(t)$. Since $T(t)$ commutes with $P^{a} \otimes I d$ for $a \subset c$, the following theorem is obtained as an immediate consequence of Proposition 5.1.

Theorem 5.2. Assume that $(V)$ is fulfilled. Then the wave operators

$$
\Omega_{a, S 1}=s-\lim _{t \rightarrow \infty} U_{c}(t)^{*} U_{a, S 1}(t)\left(P^{a} \otimes I d\right)
$$

exist for all $a \subset c$, and are asymptotically complete, that is,

$$
L^{2}(X)=\sum_{a \subset c} \oplus \text { Range } \Omega_{a, S 1} .
$$

We also need the following lemmas to analyze the propagators $\tilde{U}_{a D}(t)$ and $U_{a, S 1}(t)$.

Lemma 5.3. Let $\psi \in \mathscr{S}(X)$. Then as $t \rightarrow \infty$,

(1) $\left\|\left(D_{a}-t E\right) \tilde{U}_{a D}(t) \psi\right\|=O(1)$,

(2) $\left\|\left(D_{a}-t E\right) U_{a, S 1}(t) \psi\right\|=O(1)$.

Proof. Since

$$
\mathbf{D}_{\tilde{H}_{a D}(t)}\left(D_{a}-t E\right)=0, \quad \mathbf{D}_{H_{a, S 1}(t)}\left(D_{a}-t E\right)=0,
$$

(1) and (2) are obtained by integration.

Lemma 5.4. Let $\psi \in \mathscr{S}(X)$ and $q_{c}(x) \in S_{0}(X)$ be as in Sect. 4. Set $\phi_{c}(t, x)=$ $q_{c}\left(x / t^{2}\right)$ for $t \geqq 1$. Then as $t \rightarrow \infty$,

(1) $\left\|\left(1-\phi_{c}\left(t, 0, t D_{a}-t^{2} E / 2\right)\right) \tilde{U}_{a D}(t) \psi\right\|=O\left(t^{-1}\right)$,

(2) $\left\|\left(1-\phi_{c}\left(t, 0, t D_{a}-t^{2} E / 2\right)\right) U_{a, S 1}(t) \psi\right\|=O\left(t^{-1}\right)$.

Proof. Since $\phi_{c}\left(t, t^{2} E / 2\right) \equiv 1$ for $t \geqq 1$ and $\left|\nabla \phi_{c}(t, x)\right| \leqq C t^{-2}$, the lemma follows from Lemma 5.3.

By these lemmas, we have the next proposition.

Proposition 5.5. There exist the strong limits

$$
s-\lim _{t \rightarrow \infty} \tilde{U}_{a D}(t)^{*} U_{a, S 1}(t), \quad s-\lim _{t \rightarrow \infty} U_{a, S 1}(t)^{*} \tilde{U}_{a D}(t) .
$$

Proof. We write $\tilde{\phi}_{a}(t)=\phi_{c}\left(t, 0, t D_{a}-t^{2} E / 2\right)$. By Lemma 5.4, we have only to prove the existence of the limits

$$
\begin{aligned}
& \lim _{t \rightarrow \infty} \tilde{U}_{a D}(t)^{*} \tilde{\phi}_{a}(t) U_{a, S 1}(t) \psi, \\
& \lim _{t \rightarrow \infty} U_{a, S 1}(t)^{*} \tilde{\phi}_{a}(t) \tilde{U}_{a D}(t) \psi
\end{aligned}
$$


for $\psi \in \mathscr{S}(X)$. Since

$$
\begin{aligned}
& \frac{d}{d t} \tilde{\phi}_{a}(t)+i\left\{\tilde{H}_{a D}(t) \tilde{\phi}_{a}(t)-\tilde{\phi}_{a}(t) H_{a, S 1}(t)\right\} \\
& =-t^{-2}\left\langle\left(D_{a}-t E\right),\left(\nabla_{a} q_{c}\right)\left(t^{-1} D_{a}-E / 2\right)\right\rangle,
\end{aligned}
$$

we have the proposition by virtue of Lemma 5.3.

We replace $\tilde{U}_{a D}(t)$ by the propagator $U_{a D}(t)$ defined by (1.2). We need the following proposition.

Proposition 5.6. There exist the strong limits

$$
s-\lim _{t \rightarrow \infty} U_{a D}(t)^{*} \tilde{U}_{a D}(t), \quad s-\lim _{t \rightarrow \infty} \tilde{U}_{a D}(t)^{*} U_{a D}(t) .
$$

Proof. Noting that $I_{a}\left(s D_{a}\right)=I_{a}\left(s D_{a, \perp}\right)$, we have only to prove that as $t \rightarrow \infty$, $\int_{0}^{t}\left\{I_{c}\left(s p_{a}+s^{2} E / 2\right)-I_{c}\left(s p_{a, \perp}+s^{2} E / 2\right)\right\} d s$ converges locally uniformly in $p_{a}$. We write

$$
I_{c}\left(s p_{a}+s^{2} E / 2\right)-I_{c}\left(s p_{a, \perp}+s^{2} E / 2\right)=\int_{0}^{1}\left\langle\left(\partial_{\|} I_{c}\right)\left(\tau s p_{\|}+s p_{a, \perp}+s^{2} E / 2\right), s p_{\|}\right\rangle d \tau .
$$

We note that

$$
\begin{aligned}
\partial_{s}\left\{I_{c}\left(\tau s p_{\|}+s p_{a, \perp}+s^{2} E / 2\right)\right\}= & \left\langle\left(\partial_{\|} I_{c}\right)\left(\tau s p_{\|}+s p_{a, \perp}+s^{2} E / 2\right), \tau p_{\|}+s E\right\rangle \\
& +\left\langle\left(\partial_{\perp} I_{c}\right)\left(\tau s p_{\|}+s p_{a, \perp}+s^{2} E / 2\right), p_{a, \perp}\right\rangle \\
= & \left\langle\left(\partial_{\|} I_{c}\right)\left(\tau s p_{\|}+s p_{a, \perp}+s^{2} E / 2\right), s E\right\rangle+O\left(s^{-(1+\rho)}\right)
\end{aligned}
$$

holds locally uniformly in $p_{a}$ and uniformly in $0 \leqq \tau \leqq 1$. Here we used the fact that $\left(\nabla I_{c}\right)\left(\tau s p_{\|}+s p_{a, \perp}+s^{2} E / 2\right)=O\left(s^{-(1+\rho)}\right)$ holds locally uniformly in $p_{a}$ and uniformly in $0 \leqq \tau \leqq 1$. Hence we have

$$
\begin{aligned}
& \left\langle\left(\partial_{\|} I_{c}\right)\left(\tau s p_{\|}+s p_{a, \perp}+s^{2} E / 2\right), s p_{\|}\right\rangle \\
& \quad=\frac{\left\langle p_{\|}, \omega\right\rangle}{|E|} \partial_{s}\left\{I_{c}\left(\tau s p_{\|}+s p_{a, \perp}+s^{2} E / 2\right)\right\}+O\left(s^{-(1+\rho)}\right) .
\end{aligned}
$$

This implies the proposition.

Combining the above two propositions, we have the following proposition.

Proposition 5.7. There exist the strong limits

$$
s-\lim _{t \rightarrow \infty} U_{a D}(t)^{*} U_{a, S 1}(t), \quad s-\lim _{t \rightarrow \infty} U_{a, S 1}(t)^{*} U_{a D}(t) .
$$

We should note that these operators commute with $P^{a} \otimes I d$. Indeed, we decompose $H_{a D}(t)$ and $H_{a, S 1}$ on $L^{2}(X)=L^{2}\left(X^{a}\right) \otimes L^{2}\left(X_{a}\right)$ into

$$
H_{a D}(t)=H^{a} \otimes I d+I d \otimes T_{a D}(t), \quad H_{a, S 1}(t)=H^{a} \otimes I d+I d \otimes T_{a, S 1}(t),
$$


where

$$
\begin{gathered}
T_{a D}(t)=T_{a}+I_{a}^{c}\left(t D_{a, \perp}\right)+I_{c}\left(0, t D_{a, \perp}+t^{2} E / 2\right), \\
T_{a, S 1}(t)=T_{a}+I_{a}^{c}\left(t D_{a}\right)+W_{c}\left(t, 0, t D_{a}-t^{2} E / 2\right) .
\end{gathered}
$$

Thus, if we denote by $\hat{U}_{a D}(t)$ and $\hat{U}_{a, S 1}(t)$ the propagators which are generated by $T_{a D}(t)$ and $T_{a, S 1}(t)$, respectively, we see that

$$
\begin{aligned}
& s-\lim _{t \rightarrow \infty} U_{a D}(t)^{*} U_{a, S 1}(t)=I d \otimes\left(s-\lim _{t \rightarrow \infty} \hat{U}_{a D}(t)^{*} \hat{U}_{a, S 1}(t)\right), \\
& s-\lim _{t \rightarrow \infty} U_{a, S 1}(t)^{*} U_{a D}(t)=I d \otimes\left(s-\lim _{t \rightarrow \infty} \hat{U}_{a, S 1}(t)^{*} \hat{U}_{a D}(t)\right) .
\end{aligned}
$$

Completion of the proof of Theorem 1.1. The existence of the modified wave operators $W_{a D}^{+}$with $a \subset c$ is proved by Theorem 4.5, Theorem 5.2 and Proposition 5.7. It follows from Theorem 5.2 that Range $W_{a D}^{+} \perp$ Range $W_{b D}^{+}$if $a \neq b$.

We shall prove the asymptotic completeness. Let $\psi \in L^{2}(X)$. By Theorem 4.6, Theorem 5.2 and Proposition 5.7, we have as $t \rightarrow \infty$,

$$
\begin{aligned}
\exp (-i t H) \psi & =U_{c}(t) \psi_{c}+o(1)=\sum_{a \subset c} U_{c}(t) \Omega_{a, S 1} \psi_{c}^{a}+o(1) \\
& =\sum_{a \subset c} U_{a, S 1}(t) \psi_{c}^{a}+o(1)=\sum_{a \subset c} U_{a D}(t) \tilde{\psi}_{c}^{a}+o(1)
\end{aligned}
$$

for some $\psi_{c}^{a} \in$ Range $\left(P^{a} \otimes I d\right)$, where $\tilde{\psi}_{c}^{a}=\lim _{t \rightarrow \infty} U_{a D}(t)^{*} U_{a, S 1}(t) \psi_{c}^{a}$. This implies

$$
\psi \in \sum_{a \subset c} \oplus \text { Range } W_{a D}^{+}
$$

which completes the proof of Theorem 1.1.

\section{Case of Non-Zero Reduced Charges}

In this section, we assume that no subsystems have zero reduced charges. Then $E^{a} \neq 0$ for all cluster decompositions $a$ with $\#(a) \neq N$, and hence the maximal element $c$ of the set $\left\{a \in \mathscr{A}: E^{a}=0\right\}$ becomes the $N$-cluster decomposition. Throughout the section, $V_{\alpha}\left(x^{\alpha}\right)$ is assumed to fulfill the assumption $(V)$ :

$$
\partial_{x^{\alpha}}^{\beta} V_{\alpha}\left(x^{\alpha}\right)=O\left(\left|x^{\alpha}\right|^{-(\rho+|\beta|) / 2}\right) \text { for some } \rho>0 .
$$

The aim here is to extend the result obtained in the case of two-particle systems (see $[\mathrm{JY}]$ and $[\mathrm{W} 1, \mathrm{~W} 2]$ ) to the case of many-particle systems.

The argument below is in principle based on the same idea as in Sigal [S]. We should note that under this assumption, Theorems 4.5 and 4.6 still hold.

We use the notation $\partial_{z}=\langle\omega, \nabla\rangle$. We construct an approximate solution to the Hamilton-Jacobi equation

$$
\partial_{t} S+|E| \partial_{z} S=|p|^{2} / 2+W_{c}\left(t, \nabla_{p} S\right)
$$


associated with $H_{c}(t)$. Without loss of generality, we assume that $1 / \rho$ is not an integer. Set $L=[1 / \rho]$, so that $(L+1) \rho>1$. We first define $K_{0}(t, p)$ by

$$
K_{0}(t, p)=|p|^{2} t / 2-|E|\langle\omega, p\rangle t^{2} / 2+|E|^{2} t^{3} / 6
$$

Then $K_{0}$ satisfies

$$
\partial_{t} K_{0}+|E| \partial_{z} K_{0}=|p|^{2} / 2
$$

We further define $K_{j}(t, p), 1 \leqq j \leqq L$, for $t \geqq 1$ inductively as the solution to

$$
\partial_{t} K_{j}+|E| \partial_{z} K_{j}=F_{j-1}(t, p), \quad K_{j}(1, p)=0,
$$

where

$$
F_{j}(t, p)=W_{c}\left(t, \sum_{m=0}^{j} \nabla_{p} K_{m}(t, p)\right)-W_{c}\left(t, \sum_{m=0}^{j-1} \nabla_{p} K_{m}(t, p)\right) .
$$

\section{Lemma 6.1.}

$$
\partial_{p}^{\beta} K_{j}(t, p)=O\left(t^{1-j \rho}\right), \quad 1 \leqq j \leqq L
$$

uniformly in $p$.

Proof. The lemma is easily verified by induction. The solution $K_{J}$ is given by

$$
K_{J}(t, p)=\int_{1}^{t} F_{j-1}(s,(s-t) E+p) d s
$$

In particular, we have

$$
K_{1}(t, p)=\int_{1}^{t} W_{c}\left(s,\left(s^{2} / 2-s t\right) E+s p\right) d s,
$$

and hence $K_{1}$ obeys the estimates by (4.3). Assume that $K_{m}, 1 \leqq m \leqq j-1$, satisfies the estimates in the lemma. Then it follows that $\partial_{p}^{\beta} F_{j-1}(t, p)=O\left(t^{-\jmath \rho}\right)$. This proves that $K_{j}$ also satisfies the desired estimates and the proof is completed.

The approximate solution $S(t, p)$ to Eq. (6.1) is now defined by

$$
S(t, p)=\sum_{j=0}^{L} K_{j}(t, p), \quad t \geqq 1 .
$$

Then we have for $t \geqq 1$,

$$
\partial_{t} S+|E| \partial_{z} S-|p|^{2} / 2-W_{c}\left(t, \nabla_{p} S\right)=-F_{L}(t, p),
$$

and hence it follows from Lemma 6.1 that

$$
\partial_{p}^{\beta}\left\{\partial_{t} S+|E| \partial_{z} S-|p|^{2} / 2-W_{c}\left(t, \nabla_{p} S\right)\right\}=O\left(t^{-(L+1) \rho}\right)
$$

uniformly in $p$. We also consider the Hamiltonian

$$
\tilde{H}_{0}(t)=H_{c}+W_{0}(t, D), \quad W_{0}(t, p)=W_{c}\left(t,\left(\nabla_{p} S\right)(t, p)\right)
$$

for $t \geqq 1$ and denote the $\tilde{U}_{0}(t)$ the propagator generated by $\tilde{H}_{0}(t)$. 
Lemma 6.2. Let the notations be as above and $\psi \in \mathscr{S}(X)$. Then as $t \rightarrow \infty$,

(1) $\left\|\left(x-\left(\nabla_{p} S\right)(t, D)\right) U_{c}(t) \psi\right\|=O(1)$,

(2) $\left\|\left(x-\left(\nabla_{p} S\right)(t, D)\right) \tilde{U}_{0}(t) \psi\right\|=O(1)$.

Proof. Let $\Phi(t)=x-\left(\nabla_{p} S\right)(t, D)$. We calculate the Heisenberg derivative $\mathbf{D}_{H_{c}(t)} \Phi(t)$. We write $H_{c}(t)=\tilde{H}_{0}(t)+W_{c}(t, x)-W_{0}(t, D)$. It follows from (6.3) that

$$
\mathbf{D}_{\tilde{H}_{0}(t)}=i\left[\tilde{H}_{0}(t), \Phi(t)\right]+\Phi^{\prime}(t)=\left(\nabla_{p} F_{L}\right)(t, D)=O\left(t^{-(L+1) \rho}\right) .
$$

Noting that $-(L+1) \rho<-1,(2)$ of the lemma is proved by this estimate. We also have

$$
i\left[W_{c}(t, x)-W_{0}(t, D), \Phi(t)\right]=O\left(t^{-1-\rho}\right) \Phi(t)+O\left(t^{-1-\rho}\right)
$$

by a simple calculus of pseudodifferential operators. Hence the Heisenberg derivative $\mathbf{D}_{H_{c}(t)} \Phi(t)$ takes the form

$$
\mathbf{D}_{H_{c}(t)} \Phi(t)=O\left(t^{-1-\rho}\right) \Phi(t)+O\left(t^{-1-\rho}\right)+O\left(t^{-(L+1) \rho}\right) .
$$

This yields

$$
\left\|\Phi(t) U_{c}(t) \psi\right\| \leqq C\left\{1+\int_{1}^{t} s^{-1-\rho}\left\|\Phi(s) U_{c}(s) \psi\right\| d s\right\},
$$

and hence (1) of the lemma follows immediately from the Gronwall inequality.

By Lemma 6.2, we have the following proposition.

Proposition 6.3. There exist the strong limits

$$
s-\lim _{t \rightarrow \infty} \tilde{U}_{0}(t)^{*} U_{c}(t), \quad s-\lim _{t \rightarrow \infty} U_{c}(t)^{*} \tilde{U}_{0}(t) .
$$

Proof. By a simple calculus of pseudodifferential operators, we have

$$
W_{c}(t, x)-W_{0}(t, D)=O\left(t^{-(1+\rho)}\right)\left(x-\left(\nabla_{p} S\right)(t, D)\right)+O\left(t^{-(1+\rho)}\right) .
$$

Combining this fact with Lemma 6.2, we have the proposition.

We define the smooth function $\hat{Y}(t, p)$ with values in $X$ by

$$
\hat{Y}(t, p)=F(t \leqq 1)\left(t p-t^{2} E / 2\right)+\{1-F(t \leqq 1)\}\left(\nabla_{p} S\right)(t, p)
$$

for $t \geqq 0$. We write $\hat{W}(t, p)=V(\hat{Y}(t, p))$, and denote by $\hat{H}_{0}(t)=H_{c}+\hat{W}(t, D)$ the time-dependent Hamiltonian and by $\hat{U}_{0}(t)$ the propagator generated by $\hat{H}_{0}(t)$. Let $Y(t, p)=\hat{Y}(t, p+t E)$ and $W(t, p)=V(Y(t, p))$. Then $\hat{U}_{0}(t)$ is explicitly represented by

$$
\hat{U}_{0}(t)=\exp \left(-i t H_{c}\right) \exp \left(-i \int_{0}^{t} W(s, D) d s\right) .
$$

Then we need the following lemma.

Lemma 6.4. Let $\psi \in \mathscr{S}(X)$. Then as $t \rightarrow \infty$,

(1) $\left\|(D-t E) \tilde{U}_{0}(t) \psi\right\|=O(1)$,

(2) $\left\|(D-t E) \hat{U}_{0}(t) \psi\right\|=O(1)$,

(3) $\left\|\left(t^{-1}\left(\nabla_{p} S\right)(t, D)-t E / 2\right) \tilde{U}_{0}(t) \psi\right\|=O(1)$,

(4) $\left\|\left(t^{-1}\left(\nabla_{p} S\right)(t, D)-t E / 2\right) \hat{U}_{0}(t) \psi\right\|=O(1)$. 
Proof. (1) and (2): Since

$$
\mathbf{D}_{\tilde{H}_{0}(t)}(D-t E)=0, \quad \mathbf{D}_{\hat{H}_{0}(t)}(D-t E)=0,
$$

the statements follow by integration.

(3) and (4): We set $\Phi(t)=t^{-1}\left(\nabla_{p} S\right)(t, D)-t E / 2$. We calculate the Heisenberg derivative of $\Phi(t)$ as follows:

$$
\begin{aligned}
\mathbf{D}_{\tilde{H}_{0}(t)} \Phi(t) & =-t^{-2}\left(\nabla_{p} S\right)(t, D)-E / 2+t^{-1} D+O\left(t^{-(1+\rho)}\right) \\
& =-t^{-2} \sum_{j=1}^{L}\left(\nabla_{p} K_{j}\right)(t, D)+O\left(t^{-(1+\rho)}\right) \\
& =O\left(t^{-(1+\rho)}\right),
\end{aligned}
$$

where we used (6.3), the fact $\left(\nabla_{p} K_{0}\right)(t, D)=t D-t^{2} E / 2$ and Lemma 6.1. Similarly, we have $\mathbf{D}_{\hat{H}_{0}(t)} \Phi(t)=O\left(t^{-(1+\rho)}\right)$. Hence, the statements are proved by integration.

Lemma 6.5. Let $\psi \in \mathscr{S}(X)$ and $q_{c}(x) \in S_{0}(X)$ be as in Sect. 4. Set $\phi_{c}(t, x)=$ $q_{c}\left(x / t^{2}\right)$ for $t \geqq 1$ and write $\phi(t, p)=\phi_{c}\left(t,\left(\nabla_{p} S\right)(t, p)\right)$. Then as $t \rightarrow \infty$,

(1) $\left\|(1-\phi(t, D)) \tilde{U}_{0}(t) \psi\right\|=O\left(t^{-1}\right)$,

(2) $\left\|(1-\phi(t, D)) \hat{U}_{0}(t) \psi\right\|=O\left(t^{-1}\right)$.

Proof. Since $\phi_{c}\left(t, t^{2} E / 2\right) \equiv 1$ for $t \geqq 1$ and $\left|\nabla \phi_{c}(t, x)\right| \leqq C t^{-2}$, the lemma follows from Lemma 6.4.

By these lemmas, we have the next proposition.

Proposition 6.6. There exist the strong limits

$$
s-\lim _{t \rightarrow \infty} \hat{U}_{0}(t)^{*} \tilde{U}_{0}(t), \quad s-\lim _{t \rightarrow \infty} \tilde{U}_{0}(t)^{*} \hat{U}_{0}(t) .
$$

Proof. By Lemma 6.5, we have only to prove the existence of the limits

$$
\begin{gathered}
\lim _{t \rightarrow \infty} \hat{U}_{0}(t)^{*} \phi(t, D) \tilde{U}_{0}(t) \psi, \\
\lim _{t \rightarrow \infty} \tilde{U}_{0}(t)^{*} \phi(t, D) \hat{U}_{0}(t) \psi
\end{gathered}
$$

for $\psi \in \mathscr{S}(X)$. We should note that $\hat{Y}(t, p)=\left(\nabla_{p} S\right)(t, p)$ for $t \geqq 2$. We write $\phi^{\prime}(t, p)=\left(\nabla q_{c}\right)\left(t^{-2}\left(\nabla_{p} S\right)(t, p)\right)$. Using $(6.3)$, the fact $\left(\nabla_{p} K_{0}\right)(t, D)=t D-t^{2} E / 2$ and Lemma 6.1 , we have for $t \geqq 2$,

$$
\begin{aligned}
\frac{d}{d t} & \phi(t, D)+i\left\{\hat{H}_{0}(t) \phi(t, D)-\phi(t, D) \tilde{H}_{0}(t)\right\} \\
& =\left\langle\phi^{\prime}(t, D),\left\{-2 t^{-3}\left(\nabla_{p} S\right)(t, D)+t^{-2} D\right\}\right\rangle+O\left(t^{-(2+\rho)}\right) \\
& =\left\langle\phi^{\prime}(t, D),\left\{-t^{-2}(D-t E)-2 t^{-3} \sum_{j=1}^{L}\left(\nabla_{p} K_{j}\right)(t, D)\right\}\right\rangle+O\left(t^{-(2+\rho)}\right) \\
& =\left\langle\phi^{\prime}(t, D),\left\{-t^{-2}(D-t E)\right\}\right\rangle+O\left(t^{-(2+\rho)}\right) .
\end{aligned}
$$

Hence, we have the proposition by virtue of Lemma 6.4 . 
We define the time-dependent Hamiltonian by $H_{0}(t)=H_{c}+V\left(Y\left(t, D_{c, \perp}\right)\right)$, and denote by $U_{0}(t)$ the propagator generated by $H_{0}(t)$. Then $U_{0}(t)$ is explicitly represented by

$$
U_{0}(t)=\exp \left(-i t H_{c}\right) \exp \left(-i \int_{0}^{t} W\left(s, D_{c, \perp}\right) d s\right) .
$$

We replace $\hat{U}_{0}(t)$ by $U_{0}(t)$. We need the following proposition.

Proposition 6.7. There exist the strong limits

$$
s-\lim _{t \rightarrow \infty} U_{0}(t)^{*} \hat{U}_{0}(t), \quad s-\lim _{t \rightarrow \infty} \hat{U}_{0}(t)^{*} U_{0}(t) .
$$

Proof. Noting that $D=D_{c}$, we have only to prove that as $t \rightarrow \infty, \int_{0}^{t}\left\{W\left(s, p_{c}\right)-\right.$ $\left.W\left(s, p_{c, \perp}\right)\right\} d s$ converges locally uniformly in $p_{c}$. We write

$$
W\left(s, p_{c}\right)-W\left(s, p_{c, \perp}\right)=\int_{0}^{1}\left(\partial_{z} W\right)\left(s, \tau p_{\|}+p_{c, \perp}\right)\left\langle p_{\|}, \omega\right\rangle d \tau
$$

We note that $\partial_{t}\{Y(t, p)\}=\left(\partial_{t} \hat{Y}\right)(t, p+t E)+|E|\left(\partial_{z} \hat{Y}\right)(t, p+t E)=|E|\left(\partial_{z} \hat{Y}\right)(t, p+$ $t E)+p+O\left(t^{-\rho}\right)$ as $t \rightarrow \infty$ uniformly in $p$, by the definition of $Y(t, p)$. Then we see that as $s \rightarrow \infty$,

$$
\begin{aligned}
& \partial_{s}\left\{W\left(s, \tau p_{\|}+p_{c, \perp}\right)\right\}=\left\langle(\nabla V)\left(Y\left(s, \tau p_{\|}+p_{c, \perp}\right)\right), \partial_{s}\left\{Y\left(s, \tau p_{\|}+p_{c, \perp}\right)\right\}\right\rangle \\
& =|E|\left\langle(\nabla V)\left(Y\left(s, \tau p_{\|}+p_{c, \perp}\right)\right),\left(\partial_{z} \hat{Y}\right)\left(s, \tau p_{\|}+p_{c, \perp}+s E\right)\right\rangle+O\left(s^{-(1+\rho)}\right)
\end{aligned}
$$

holds locally uniformly in $p_{c}$ and uniformly in $0 \leqq \tau \leqq 1$. Here we used the fact that $(\nabla V)\left(Y\left(s, \tau p_{\|}+p_{c, \perp}\right)\right)=O\left(s^{-(1+\rho)}\right)$ holds locally uniformly in $p_{c}$ and uniformly in $0 \leqq \tau \leqq 1$. Hence we see that

$$
\begin{aligned}
& \left(\partial_{z} W\right)\left(s, \tau p_{\|}+p_{c, \perp}\right)\left\langle p_{\|}, \omega\right\rangle \\
& =\left\langle(\nabla V)\left(Y\left(s, \tau p_{\|}+p_{c, \perp}\right)\right),\left(\partial_{z} \hat{Y}\right)\left(s, \tau p_{\|}+p_{c, \perp}+s E\right)\right\rangle\left\langle p_{\|}, \omega\right\rangle \\
& \quad=\frac{\left\langle p_{\|}, \omega\right\rangle}{|E|} \partial_{s}\left\{W\left(s, \tau p_{\|}+p_{c, \perp}\right)\right\}+O\left(s^{-(1+\rho)}\right)
\end{aligned}
$$

holds locally uniformly in $p_{c}$ and uniformly in $0 \leqq \tau \leqq 1$. This implies the proposition.

Combining the above two propositions, we have the following proposition.

Proposition 6.8. There exist the strong limits

$$
s-\lim _{t \rightarrow \infty} U_{0}(t)^{*} \tilde{U}_{0}(t), \quad s-\lim _{t \rightarrow \infty} \tilde{U}_{0}(t)^{*} U_{0}(t) .
$$

What we want to prove here is the following theorem.

Theorem 6.9. Assume that $c$ is the $N$-cluster decomposition and the assumption $(V)$ is fulfilled. Then one can construct a function $Y(t, p) \in C^{\infty}(\mathbf{R} \times X)$ with 
values in $X$ such that the modified wave operators

$$
W_{0}^{ \pm}=s-\lim _{t \rightarrow \pm \infty} \exp (i t H) \exp \left(-i t H_{c}\right) \exp \left(-i \int_{0}^{t} W\left(s, D_{c, \perp}\right) d s\right)
$$

exist and are asymptotically complete, that is, $W_{0}^{ \pm}$are unitary operators on $L^{2}(X)$, where $W(t, p)=V(Y(t, p))$. Moreover, $W_{0}^{ \pm}$have the intertwining property.

Proof. We prove the theorem for $W_{0}^{+}$only. The existence of $W_{0}^{+}$is proved by Theorem 4.5, Propositions 6.3 and 6.8. Then it is easy to see that $W_{0}^{+}$has the intertwining property. By Propositions 6.3 and 6.8, we see that there exists the strong limit

$$
\tilde{\Omega}_{0}^{+}=s-\lim _{t \rightarrow \infty} U_{0}(t)^{*} U_{c}(t) .
$$

Let $\psi \in L^{2}(X)$. By Theorem 4.6 and the above fact, we have as $t \rightarrow \infty$,

$$
\exp (-i t H) \psi=U_{c}(t) \psi_{c}+o(1)=U_{0}(t) \tilde{\Omega}_{0}^{+} \psi_{c}+o(1) .
$$

This implies $\psi \in$ Range $W_{0}^{+}$, hence we have $L^{2}(X)=$ Range $W_{0}^{+}$. Noting that $W_{0}^{+}$ is an isometry, the proof of the theorem is completed.

Remark 6.10. If, in particular, the assumption $(V)$ is satisfied with $\rho>1 / 2$, then we can take $Y(t, p)$ as $Y(t, p)=t p+t^{2} E / 2$, that is, we may take $W_{0}^{+}$as the Dollardtype modified wave operators (1.3).

Acknowledgements. T. Adachi thanks Professor Kenji Yajima for many valuable discussions and suggestions.

\section{References}

[A] Adachi, T.: Long-range scattering for three-body Stark Hamiltonians. J. Math. Phys. 35, 5547-5571 (1994)

[AT] Adachi, T., Tamura, H.: Asymptotic Completeness for Long-Range Many-Particle Systems with Stark Effect. To appear in J. Math. Sci., The Univ. of Tokyo

[AH] Avron, J.E., Herbst, I.W.: Spectral and scattering theory of Schrödinger operators related to the Stark effect. Commun. Math. Phys. 52, 239-254 (1977)

[D] Dereziński, J.: Asymptotic completeness of long-range $N$-body quantum systems. Ann. Math. 138, 427-476 (1993)

[E] Enss, V.: Long-range scattering of two- and three-body systems. Proceedings of the Conference 'Equations aux dérivées partielles' Saint Lean de Monts, Ecole Polytechnique 1989, pp. $1-31$

[G] Gérard, C.: Asymptotic completeness for 3-particle long-range systems. Invent. Math. 114, 333-397 (1993)

[Gr1] Graf, G.M.: Asymptotic completeness for $N$-body short-range quantum systems: A new proof. Commun. Math. Phys. 132, 73-101 (1990)

[Gr2] Graf, G.M.: A remark on long-range Stark scattering. Helv. Phys. Acta 64, 1167-1174 (1991)

[HeSj] Helffer, B., Sjöstrand, J.: Equation de Schrödinger avec champ magnétique et équation de Harper. Lecture Notes in Physics 345, Berlin-Heidelberg-New York: Springer-Verlag, 1989 , p. $118-197$

[HS] Herbst, I.W., Skibsted, E.: Spectral analysis of $N$-body Stark Hamiltonians (smooth case). Preprint, 1993 
[JO] Jensen, A., Ozawa, T.: Existence and non-existence results for wave operators for perturbations of the Laplacian. Rev. Math. Phys. 5, 601-629 (1993)

[JY] Jensen, A., Yajima, K.: On the long-range scattering for Stark Hamiltonians. J. reine angew. Math. 420, 179-193 (1991)

[Ki] Kitada, H.: Asymptotic completeness of $N$-body wave operators I. Short-range quantum systems. Rev. Math. Phys. 3, 101-124 (1991)

[Mø] Møller, J.S.: Asymptotic completeness for $N$-body short-range Stark Hamiltonians. Preprint, 1993

[O] Ozawa, T.: Non-existence of wave operators for Stark effect Hamiltonians. Math. Z. 207, 335-339 (1991)

[S] Sigal, I.M.: On long-range scattering. Duke Math. J. 60, 473-496 (1990)

[SS1] Sigal, I.M., Soffer, A.: The $N$-particle scattering problem: Asymptotic completeness for short-range systems. Ann. Math. 125, 35-108 (1987)

[SS2] Sigal, I.M., Soffer, A.: Long-range many body scattering: Asymptotic clustering for Coulomb type potentials. Invent. Math. 99, 115-143 (1990)

[Sk] Skibsted, E.: Absolute spectral continuity for $N$-body Stark Hamiltonians. Preprint, 1993

[T1] Tamura, H.: Asymptotic completeness for $N$-body Schrödinger operators with short-range interactions. Commun. Partial Differ. Eqs. 16, 1129-1154 (1991)

[T2] Tamura, H.: Spectral and scattering theory for 3-particle Hamiltonian with Stark effect: Asymptotic completeness. Osaka. J. Math. 29, 135-159 (1990)

[T3] Tamura, H.: Scattering theory for $N$-particle Systems with Stark effect: Asymptotic Completeness. Publ. RIMS Kyoto Univ. 29, 869-884 (1993)

[Wa] Wang, X.P.: On the Three-Body Long-Range Scattering Problems. Lett. Math. Phys. 25, 267-276 (1992)

[W1] White, D.: The Stark effect and long-range scattering in two Hilbert spaces. Indiana Univ. Math. J. 39, 517-546 (1990)

[W2] White, D.: Modified wave operators and Stark Hamiltonians. Duke Math. J. 68, 83-100 (1992)

[Y] Yafaev, D.: Radiation conditions and scattering theory for $N$-particle Hamiltonians. Commun. Math. Phys. 154, 523-554 (1993)

[Z] Zielinski, L.: A proof of asymptotic completeness for $N$-body Schrödinger operators. Commun. Partial Differ. Eqs. 19, 455-522 (1994)

[Zo] Zorbas, J.: Scattering theory for Stark Hamiltonians involving long-range potentials. J. Math. Phys. 19, 577-580 (1978) 
